\title{
It's a Non-Dialysis Day... Do You Know How Your Patient Is Doing? A Case for Research into Interdialytic Activity
}

\author{
Melissa Mendoza ${ }^{\mathrm{a}, \mathrm{c}}$ Maggie Han ${ }^{\mathrm{a}}$ Anna Meyring-Wösten ${ }^{\mathrm{a}}$ Kenneth Wilund $^{\mathrm{b}}$ \\ Peter Kotanko ${ }^{a}$ \\ ${ }^{a}$ Renal Research Institute, New York, N.Y., b Department of Kinesiology and Community Health, College of Applied \\ Health Sciences, University of Illinois at Urbana-Champaign, Champaign, III., and 'Department of Biomedical \\ Engineering, Rochester Institute of Technology, Rochester, N.Y., USA
}

\section{Key Words}

Interdialytic · Physical activity · Hemodialysis · Physical function - Exercise - CKD · ESRD - Tracking devices - Exercise training in HD patients

\begin{abstract}
Hemodialysis (HD) patients are less active than their healthy counterparts; this is associated with higher mortality. Healthcare workers observe their patients only during HD, which accounts for about $7 \%$ of the week. Knowing more about what occurs in between sessions, particularly with respect to physical activity, may improve patient care and prognosis. Yet without a standard method to measure interdialytic activity, it is difficult to compare the effect of interventions. However, it is unclear how interdialytic activity can be accurately measured. Since activity associated with quality of life is multi-dimensional, objective and subjective tools should be used in conjunction. While commercially available tracking devices can be seamlessly incorporated into everyday life and can increase awareness of user's activity, their validation is needed in the HD population. Fertile topics for research should include the relationship between objective and subjective measures in HD patients, and the investigation of physical activity in non-ambulatory HD patients.
\end{abstract}

(c) 2015 S. Karger AG, Basel

0253-5068/15/0393-0074\$39.50/0

\section{Introduction}

Physical activity is any body movement produced by skeletal muscles that requires energy expenditure [1]. According to World Health Organization (WHO), physical inactivity is identified as the fourth leading cause of global mortality [2]. A recent study in patients with impaired glucose tolerance indicated that per 2,000 steps per day cardiovascular events were reduced by $10 \%$ [3]. In the chronic kidney disease (CKD) population, leading a nonsedentary lifestyle is associated with a lower mortality risk [4]. It has also been found that hemodialysis (HD) patients who are more physically active experience less postdialysis fatigue, have higher cognitive performance, and are less likely to report symptoms of depression and insomnia $[5,6]$. While for some patients, leading a sedentary lifestyle is unavoidable due to physical impediments such as muscle atrophy or excessive fatigue, others avoid physical activity due to lack of motivation or support and a feeling of helplessness [7-9]. For patients who are physically able to be more active, interventions can be implemented to encourage physical activity. In order to detect the effectiveness of an intervention, reliable and repeat-

M.M. and M.H. contributed equally to this work.

\section{KARGER 125}

E-Mail karger@karger.com www.karger.com/bpu
Peter Kotanko, MD

Renal Research Institute 315 East 62nd Street, 4th Floor

New York, NY 10065 (USA)

E-Mail pkotanko@ rriny.com 
able tools, and/or methods by which to measure physical activity are necessary.

The KDIGO (Kidney Disease: Improving Global Outcomes) guideline recommends an $\mathrm{HD}$ patient to exercise for at least $30 \mathrm{~min}$, five times a week [10]. However, this guideline does not consider the different stages of CKD or the individual needs of patients. Though many physicians recognize the importance of physical activity, it has been cited that some physicians are mistaken in believing that patients are not interested in becoming more physically active [11]. Furthermore, healthcare workers may feel unsure of how to encourage their patients $[11,12]$. It has been recommended that physicians should be given more tools, with which they can advise patients to improve physical activity [11].

The use of new technology and programs that track and motivate people to be more physically active has been implemented into research, especially for those patients with chronic diseases and comorbidities. Likewise, renal research has started to implement the use of these types of devices and programs to assess the physical activity of HD patients during their daily lives. This review focuses on interdialytic physical activity, and the advancements in tracking tools that might be useful in the field of kidney research.

\section{Intradialytic Exercise Training}

Dozens of studies published in the past $20-30$ years have documented the benefits of exercise training in HD patients [13-16]. A majority of these studies have utilized exercise during HD treatment (intradialytic exercise), which is often preferred for a variety of reasons, including reducing patient burden and improving exercise compliance [17]. Similar to exercise in other patient populations, exercise training in HD patients, in particular during dialysis treatment, has been shown to improve traditional cardiovascular disease (CVD) risk factors (e.g., plasma lipid profiles, blood pressure), and various metrics related to physical function (e.g., aerobic capacity/ $/ \mathrm{VO}_{2} \mathrm{max}$ ). While the data from these studies is impressive in many regards, there are also significant shortcomings, as many were pilot studies with a relatively small number of subjects, the exercise interventions were often short (e.g., 2-3 months), and very few contained randomized control groups. More recently, several longer-term randomized controlled trials (RCTs) examining the efficacy of intradialytic endurance and or resistance exercise training in HD patients have been published [18-23]. The training protocols in these studies included intradialytic cycling or low-load resistance training using bands and ankle weights. While some improvements in physical function and reductions in CVD risk were found in these studies, much of the data is inconsistent, or in some cases even negative. Indeed, there is ongoing research exploring potential mechanisms responsible for the putative lack of efficacy of exercise training in HD patients [24]. These equivocal findings may also partially explain the low rates of exercise counseling by nephrologists to their patients [25]. A potential reason for these inconsistent benefits is that there are clear limits to the volume, intensity, and types of exercise protocols that can be performed while patients are confined to their chair or bed during treatment. While intradialytic exercise can be an important component of patients' care plan, it is critical to find ways to increase physical activity in between dialysis sessions (interdialytic activity) to maximize their health.

\section{Significance of Interdialytic Physical Activity}

For the most part, healthcare workers can observe HD patients only during their dialysis sessions. These sessions usually take place three times a week for $3-5 \mathrm{~h}$ at a time. However, during the other $150+\mathrm{h}$ of the week, physicians know little about patient activity. In other words, physicians and healthcare workers observe patients only for less than $10 \%$ of the week. Tracking a patient in between dialysis treatments will give a more comprehensive understanding of overall physical activity.

\section{Tools to Assess Interdialytic Physical Activity}

To our knowledge, there exists no standard method for measuring either habitual activity levels or physical function. Self-reported physical activity questionnaires are often used to estimate activity levels, but are generally unreliable due to their subjective nature. A variety of objective physical performance tests can be used to assess function. However, these tests are often difficult to perform on a routine basis at the clinic due to lack of either space or equipment, and some can be impractical for CKD patients with comorbidities that reduce muscle strength. Moreover, by definition, physical performance tests only assess functional ability on a specific task, so they cannot be used to estimate a patient's habitual physical activity levels. This is important, as physical activity levels are independently related to morbidity and mortal- 
Table 1. Tools used for assessing physical activity and function discussed in this review

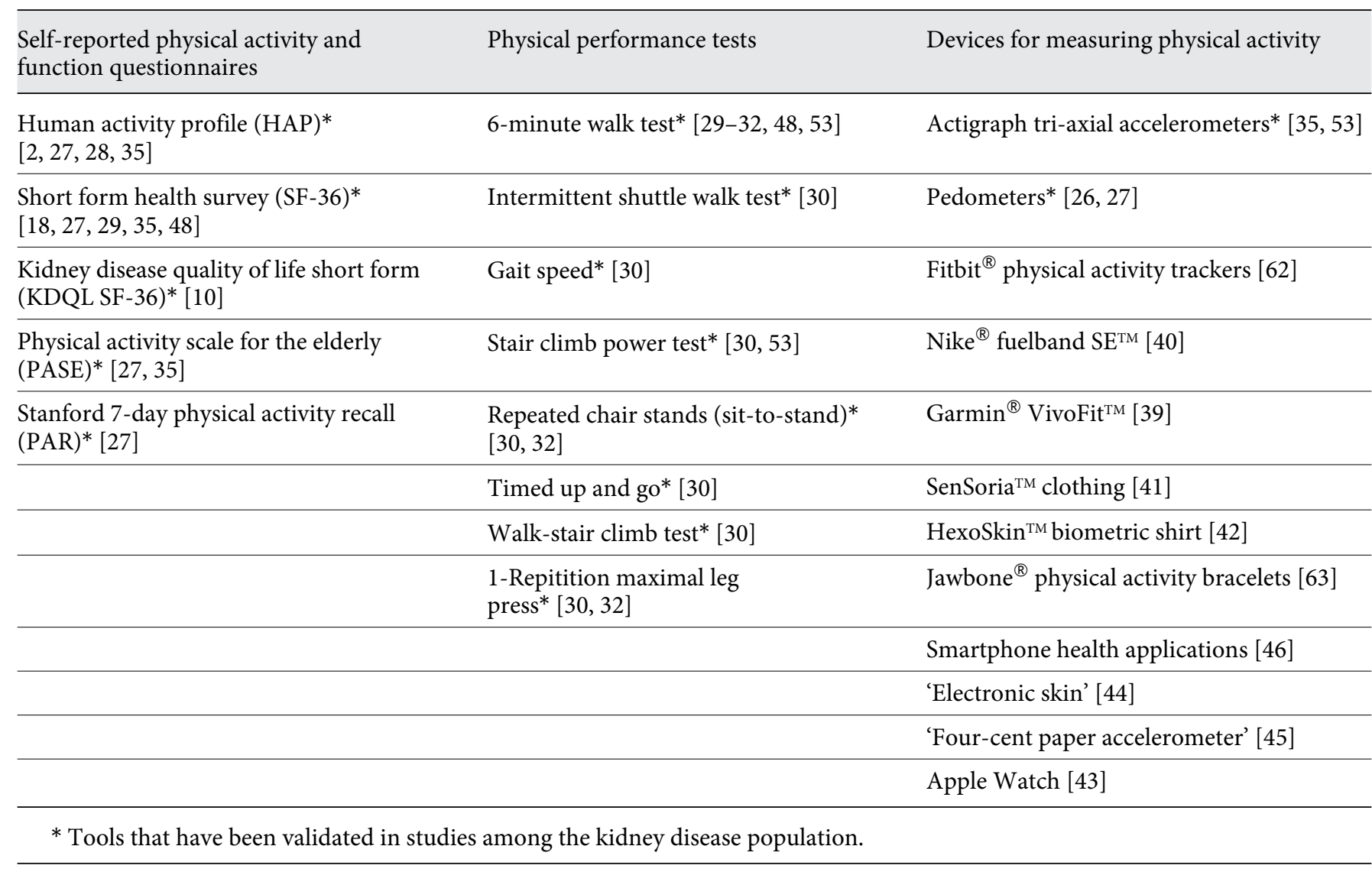

ity [4]. Fortunately, there are a number of devices such as pedometers and tri-axial accelerometers (table 1) that may be used to monitor interdialytic physical activity [26]. The relative merits of these different tools will be discussed in more detail below.

\section{Self-Reported Questionnaires}

Self-reported questionnaires may be used to assess the physical functioning of HD patients. One example is the human activity profile (HAP), which has been shown to be effective because it covers a large range of activities from 'getting in and out of chairs or bed without assistance' to 'running or jogging 3 miles in 30 min or less'. This questionnaire is most commonly used in longitudinal or interventional studies relating to physical functioning [27]. Johansen et al. utilized the HAP to establish an association of lower maximal and adjusted activity scores (MAS and AAS) with poor physical function and mental health [28]. Lower AAS was also associated with more post-dialysis fatigue [6]. Another validated question- naire, Short Form Health Survey (SF-36), measures a patient's health status consisting of eight-scaled scores varying from topics like 'vitality' to 'mental health'. Malagoni et al. utilized the SF-36 to assess the acute and long-term effects of an exercise program for dialysis patients both in a hospital and at home. Similarly, a recent study by Koh et al. evaluated physical functioning in HD patients through the use of the SF-36 questionnaire $[18,29]$. The Kidney Disease Quality of Life Short Form (KDQOL SF36 ) is a version of the SF-36 with an additional 43 questions specifically designed for dialysis patients. Questions range from the effects and burdens of living with kidney disease to the patient's perceptions of dialysis staff encouragement [10].

Along with physical function, self-reported questionnaires may also be used to assess physical activity levels. Johansen et al. have validated both the Stanford 7-day physical activity recall questionnaire (PAR) and the physical activity scale for the elderly (PASE) in HD patients. The PAR collects information about the time spent per- 
forming various levels of activities during the previous seven days. A metabolic equivalent (MET) is then assigned to sleep, light, moderate, hard, and very hard levels of physical activity and calorie expenditure can then be estimated from MET. The PAR has been validated against doubly labeled water method (DLW), heart rate monitoring, changes in maximal oxygen uptake, and accelerometry. DLW is a noninvasive and innocuous technique by which stable isotopes are used to measure energy expenditure. This method has been described as the 'gold standard' for measuring energy expenditure during daily life. The PASE asks questions about the amount of time spent performing leisure time, household, and work-related activity. Each activity is given a weight based on a comparison made with accelerometry counts. The total score is calculated by the sum of the amount of time in each activity multiplied by the weight of the activity [27].

\section{Physical Performance Tests}

Physical performance 'field'-based examinations have been used in studies involving HD patients to measure mobility and performance capacity. These tests have been proven to show time efficiency, cost-effectiveness, reproducibility, and low patient burden. Physical performance tests are useful prognostic tools as well [30]. It was observed that survival increased approximately $5 \%$ for every $100 \mathrm{~m}$ walked in the six-minute walk test (6-MWT) [31].

Physical performance tests are often used in conjunction with other measures to assess a correlation between physical activity levels and physical function. A recent study by Majchrzak et al. analyzed physical activity and function patterns in chronic HD patients using tri-axial accelerometers. Measures of physical function, including sit-to-stand test, 6-MWT, and 1-repitition maximal leg press strength were correlated with activity levels on both dialysis and non-dialysis days. It was concluded that patients are $24 \%$ less active on dialysis days when compared with non-dialysis days [32].

\section{Devices}

Advancing technologies in accelerometry and physical activity trackers may give improved tools to examine changes in interdialytic physical activity levels, and how they may be related to outcomes in HD patients. While HD patients are characterized by low physical activity and low physical function, little is known about the ways in which physicians and healthcare workers can motivate their patients to become more active [33]. Pedometers and some advanced tri-axial accelerometers such as the
Actigraph have been used to track activity in research studies for many years. Although pedometers are frequently used in studies focused on analyzing physical activity and performance, they can often be unreliable [34]. Actigraph tri-axial accelerometers have also been used in studies involving the HD population. In a study by Robinson-Cohen et al., a combination of measurement tools was used to account for physical activity counts in patients with CKD. It was concluded that questionnaire scores were significantly associated with physical activity counts [35]. Actigraph tri-axial accelerometers are reliable; however, due to their bulkiness, patients are likely to be noncompliant, and not wear them.

Recently, a variety of more user-friendly tracking devices has been developed and is commercially available. An incomplete list of examples includes Fitbit ${ }^{\circledR}, \mathrm{Nike}^{\circledR}$, Garmin ${ }^{\circledR}$, HexoSkin ${ }^{\mathrm{TM}}$, and Jawbone ${ }^{\circledR}$. These new devices have made it easier for users to track their own daily physical activity. These devices measure the user's fitness not only in the form of steps, active minutes, calories burned, but also in terms of hours and quality of sleep, among other factors. The data gathered using a 3-axis accelerometer is analyzed by different algorithms using information such as height, weight, and age provided by the user. These commercially available products have also found their way into medical research studies [17, 26, 32, 36-38].

Monitoring bracelets such as the Garmin ${ }^{\circledR}$ VivoFit ${ }^{\mathrm{TM}}$ and the Nike ${ }^{\circledR}$ Fuelband $\mathrm{SE}^{\mathrm{TM}}$ alert the user after an hour of inactivity $[39,40]$. SenSoria ${ }^{\mathrm{TM}}$ and HexoSkin ${ }^{\mathrm{TM}}$ have revolutionized these technologies by incorporating trackers into clothing such as shirts, socks, and sports bras that also measure the user's heart rate $[41,42]$. In addition to the heart rate, HexoSkin ${ }^{\mathrm{TM}}$ 's Biometric shirt informs users of their heart rate variability, breathing rate, and breathing volume. This device not only focuses on informing patients, but also on providing healthcare workers with patients' real-time data. This information allows the analysis of a group of patients and provides insight into their physical activity, sleep quality and other health domains. Furthermore, the recently unveiled Apple Watch demonstrates that due to popular demand, physical activity trackers are making their way into everyday wearable technology [43].

Researchers have also been developing ways in which to make these products even more user-friendly and costefficient. Products such as 'Electronic Skin' and the 'Fourcent Paper Accelerometer', although still prototypes, are accelerometers that are flexible and easy to use [44, 45]. The 'Four-cent Paper Accelerometer', developed by G. Whitesides and his research team at Harvard, utilizes 
simple products such as chromatography paper, tiny silver and carbon contact pads, and vinyl stencils to create an accelerometer that can possibly span uses from consumer electronics to medicine. These cheap microelectromechanical devices emulate piezoresistive silicon sensors that track changes in resistivity when the material undergoes mechanical stress to analyze different movements [45]. On the other hand, 'Electronic Skin', an idea pioneered by researchers at University of Illinois, contains flexible micro-scaled circuits, sensors, and other electrical components. When integrated with an accelerometer, these soft circuits have the ability to collect data about a person's motions throughout the day [44].

Another novel application is the integration of accelerometers and gyroscopes within smartphones. Incorporating the use of a smartphone's existing properties eliminates the need for additional devices. Several health applications have also used the integrated motion sensors of smartphones to provide users with information about their activity [46]. The implementation of these tracking devices has provided novel tools for researchers to track patient's physical activity [33].

\section{Interventions to Increase Interdialytic Activity and Outcomes}

Pedometers can be useful tools to inform patients how much they are walking. In a study by Nowicki et al., daily spontaneous activity of HD patients increased over a four month period after they had been given pedometers. This method was found to be useful even in patients who were not compliant with exercise programs [37]. Bohm et al. found that both an interdialytic cycling program and a home-based pedometer program resulted in similar levels of improvements to lower extremity function and flexibility over 24 weeks, but there was no significant changes to subjects' aerobic capacity [47]. Commercially available pedometers are very economical and easy to use.

Numerous studies have shown that exercise training interventions of various modes, duration, and intensity may have benefits in HD patients [16]. Interestingly, only a few published intervention studies have utilized interdialytic exercise as a primary component of the intervention. In the largest and longest exercise intervention published to date, Koh et al. evaluated the relative efficacy of home-based and clinic-based exercise for 6 months. Surprisingly, neither of the exercise protocols yielded significant benefits compared to a non-exercising control group [18]. However, several other studies have shown benefits of interdialytic exercise interventions. For example, Maligoni et al. demonstrated that patients adhering to an exercise program experienced lower post-dialysis fatigue and higher health-related quality of life (HRQL) than patients who did not adhere to an exercise program [48]. Another study utilized a pragmatic 12-week renal rehabilitation program instituted by the National Health Service (NHS). This study found that in patients who participated regularly, exercise-based rehabilitation can substantially improve overall physical function and mental well-being [49].

Although interdialytic exercise programs may have some merit, they tend to have a greater drop-out rate than intradialytic exercise programs [50]. Examination of adherence to exercise programs by HD patients found that adherent and non-adherent patients differed significantly, not just physiologically, but also psychologically. Characteristics such as age and dialysis vintage were strongly associated with adherence to the exercise program. Patients who were 41-60 years old were the most likely to be adherent. Patients with a vintage between 2 and 5 years had higher rates of adherence than patients who were out of that range. Patients with an internal locus of control were more likely to adhere to exercise programs than patients with an external locus of control. A patient with an internal locus of control believes that he or she can control life's events. On the contrary, a patient with an external locus of control believes that random external factors (e.g., chance, luck) control life's events. Additionally, membership to a support group improved adherence [7]. These findings can help physicians and physical therapists identify patients who are likely to adhere to an exercise program.

In a meta-analysis of interventions to increase physical activity among chronically ill patients (hypertension, coronary artery disease, cancers, renal disease, etc.), supervised exercise was found to be the most commonly used intervention. However, the meta-analysis revealed education/motivational sessions were just as effective as supervised exercise. This can help save costs since supervised exercise incurs substantial costs because it requires specialized equipment and highly trained personnel. It was also found that the only intervention strategy that had a substantial effect on physical activity was self-monitoring. Self-monitoring provides subjects with real-time information, and changes in their physical activity. Furthermore, a combination of various interventions such as goal setting, contracting, feedback, consequences, selfmonitoring, and/or prompts were found to be effective [51].
Mendoza/Han/Meyring-Wösten/Wilund/ Kotanko 
Fig. 1. Different measures of physical activity and function in both HD patients and healthy controls. This representation indicates in which area(s) HD patients are performing less than healthy controls. These values are shown as ratios normalized so that healthy control value is 1 for each measure. AAS = Adjusted activity score; PAR = physical activity recall; PASE = physical activity scale for the elderly; 7-d RQ EEE = 7-day recall questionnaire estimated energy expenditure.

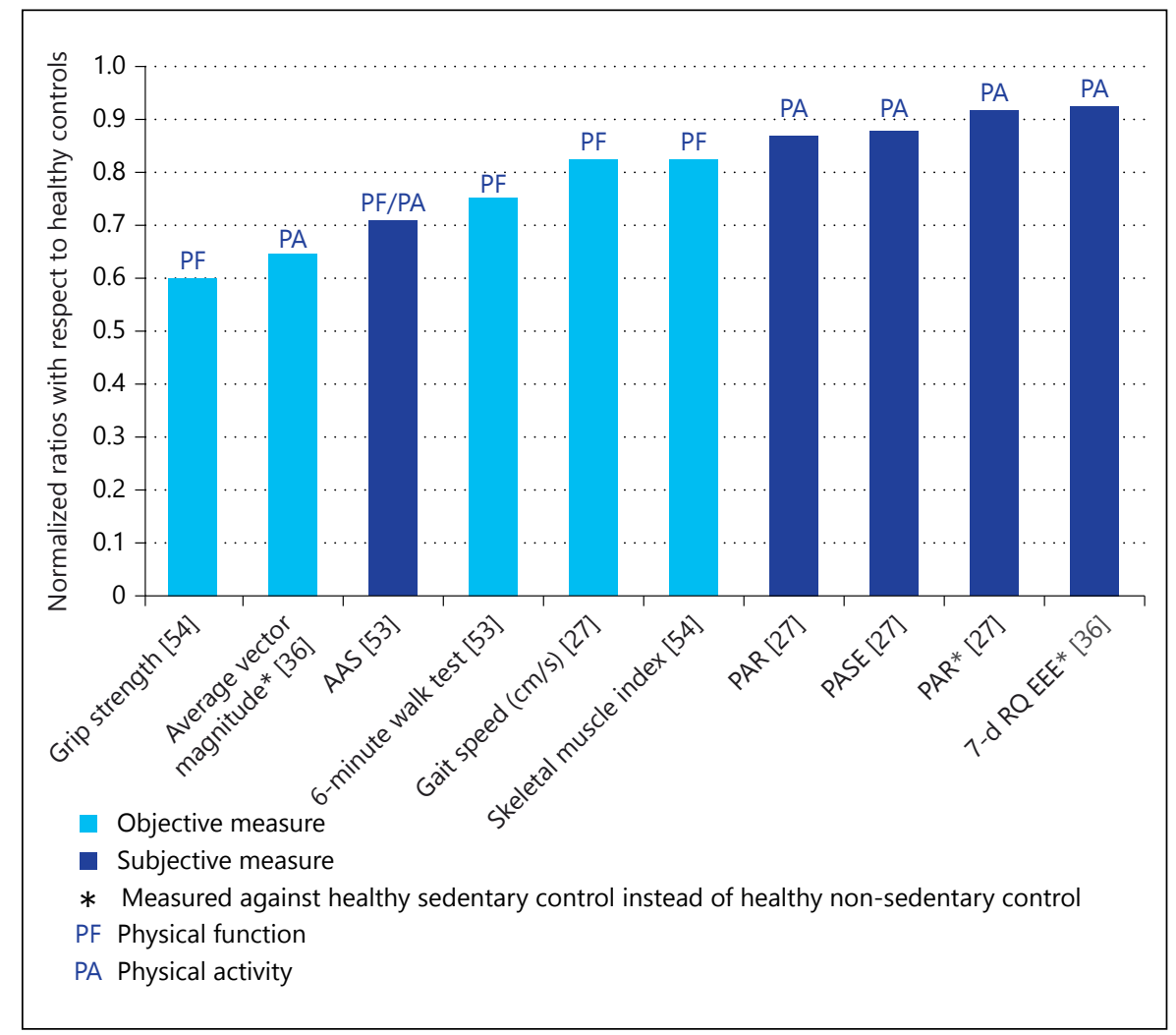

\section{Physical Activity and Function Comparison: Hemodialysis Patients versus Healthy Controls}

In current literature, most studies aim to draw conclusions through the use of different study arms, usually one with an intervention in HD patients and another without an intervention in HD patients. Some studies, however, aim to provide a comparison between the activities in $\mathrm{HD}$ patients and healthy controls. This comparison is useful for identifying the aspects of physical activity or function in which the HD population is lacking significantly. For example, an observational study by Johansen et al. determined that HD patients are less active than their non-CKD sedentary counterparts based on the vector magnitudes from accelerometers and energy expenditure values recorded [36]. Energy expenditure values describe the sum of the basal metabolic rate and the energy expended during physical activity [52]. Zhang et al. concluded that anxiety and depression are directly associated with impaired physical performance when comparing the results from a Beck Anxiety Inventory (BAI) exam, a 6-MWT, and a stair climbing test among HD and healthy controls. It was also found that HD patients who had anxiety and depression had significantly lower AAS than controls who had no anxiety and depression [53]. Another study comparing HD patients and healthy controls found a positive association between physical activity volumes and skeletal muscle mass by utilizing handgrip strength and skeletal muscle index (SMI) [54]. Hand-grip strength tests are used to observe the progression of muscle strength and are typically measured with a dynamometer [55]. Skeletal muscle index is defined by the skeletal muscle mass over body mass obtained through bioimpedance analysis. This measurement can be useful in the HD population because other studies have shown a direct relationship between reduced relative skeletal muscle mass in older individuals and functional impairment [56]. Normalized values comparing various markers of physical activity and performance between HD patients and healthy controls are shown in figure 1 . Also, the figure indicates which values measure physical activity and which describe physical function, as well as differentiates values that were gathered from subjective versus objective tools. It is evident that the difference between HD patients and controls is larger when using objective tools. 
Fig. 2. Scatter plot of correlation coefficients between direct measures and selfreported measures. Reproduced with permission from Prince et al. [59].

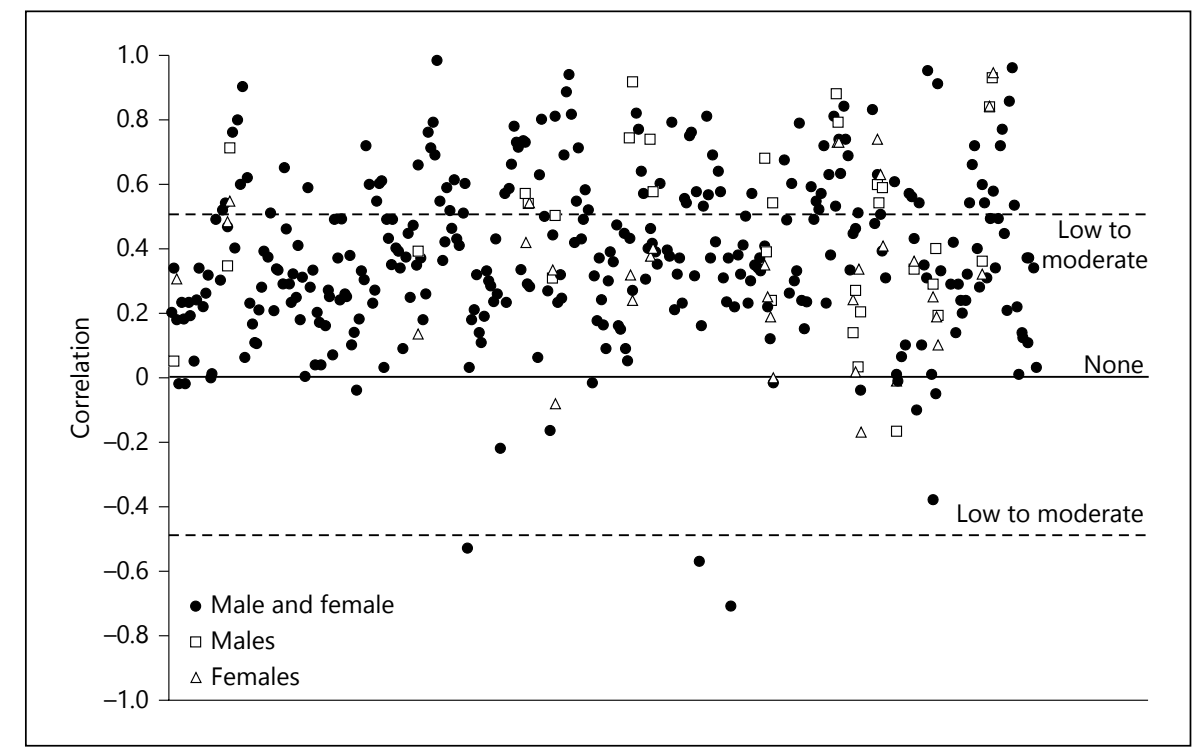

\section{Barriers to Physical Activity}

HD patients are less active than healthy individuals in part because they have physical problems which make activity difficult. Delgado et al. reported the two most common physical barriers were fatigue and shortness of breath in HD patients [57]. Diabetes, a comorbidity that may lead to amputations, is another example of a physical barrier to activity. While it is difficult to overcome these physical barriers, psychological barriers may be remedied more easily. Lack of motivation and interest are major barriers to increasing physical activity in this population [8]. Interestingly, while patient perceptions of not having sufficient time to exercise and too many medical problems were associated with low physical activity, the actual number of comorbidities was not [57]. This suggests that a patient's perception of the burden of the disease may be responsible, at least partially, for low physical activity rather than the actual disease itself.

\section{Discussion}

WHO recognizes that there are major research gaps in physical activity recommendations for patients with cardiovascular disease, diabetes, cancer, obesity, mental health conditions, and other illnesses [2]. This review focuses on how interdialytic activity is currently measured and interventions to increase it among those on HD. After examining the literature, it is evident that there are many knowledge gaps pertaining to interdialytic physical activity.

Despite a plethora of tools that are now available, how to reliably track interdialytic physical activity is still a conundrum. A solution could be to utilize easy-to-use tracking devices (Fitbit, Jawbone, etc.) in lieu of validated accelerometers, such as the Actigraph. The advantage of these next-generation tracking devices is that they are very user friendly, and can be seamlessly incorporated into daily life, whereas the Actigraph is bulky and difficult for patients to wear. While the commercially available tracking devices are not as accurate as DLW, which is the gold standard, they are far more cost efficient. Several of these tools have been validated in the non-HD population, but validating the use of the new devices in $\mathrm{HD}$ patients should be a priority so researchers can take advantage of these cost-effective and accurate tools for enhancing patient care.

One review suggested that researchers develop studies that utilize both accelerometry and DLW to accurately measure physical activity levels. DLW is very costly and is done only in studies with small populations. Therefore, developing this type of study will allow for the creation of prediction models that correlate accelerometry and subject demographics with DLW-derived energy expenditure measures [58].

Additionally, a systematic review of studies, by Prince et al., which used both self-report (subjective) and direct (objective) measures of physical activity in adults found that there is no clear relationship between the two (fig. 2).
Mendoza/Han/Meyring-Wösten/Wilund/ Kotanko 


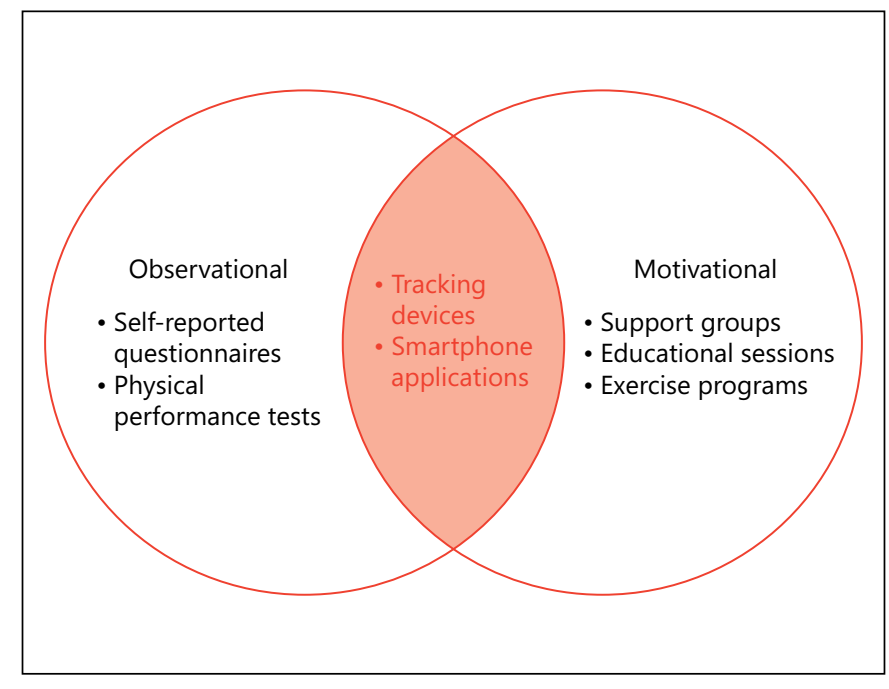

Fig. 3. Interventions used for different types of studies.

The subjective measures used in the studies reviewed were diaries/logs, questionnaires, surveys, or recall interviews. The objective measures used in the studies reviewed were DLW, indirect/direct calorimetry, pedometry, heart rate monitoring (HRM), global positioning systems, or direct observation. The mean of the correlations between self-reported and direct measures was 0.37 $(\mathrm{SD}=0.25)$ and the range was from -0.71 to 0.98 . Possible explanations for the unclear relationship between selfreport and direct measures were given. Self-report instruments may not be able to capture activities that last for $<10$ min or those with a level of exertion below a brisk walk. This would mean that in some self-report instruments, not all physical activity that is performed is reported. In addition, accelerometers are unable to capture certain physical activity such as swimming and other activities of the upper extremities, which may lead to an underestimation of physical activity [59]. The presence of over- and under-estimation in both direct and self-reported measures of physical activity makes the relationship between the two unclear.

Nevertheless, a successful attempt to calibrate self-reported physical activity was made by Saint-Maurice et al. in youths. A self-report tool called the physical activity questionnaire (PAQ) was calibrated against objectively measured physical activity utilizing the Actigraph accelerometer. The subjects wore the Actigraph accelerometer for a week and then completed the PAQ. Multiple linear regression modeling was used on $70 \%$ of the study sample to derive a calibration equation. After, this equation was cross validated in the remaining $30 \%$ of the study sample. The calibration model (equation 1), which included gender, ages, and PAQ scores, explained $40 \%$ of variance in percentage of time in moderate to vigorous physical activity (\% MVPA). When the model was tested in the remaining $30 \%$ of the sample, the portion that was not included in the multiple linear regression model, estimated $\%$ MVPA was highly correlated to actual accelerometer values. Also, the difference between the estimated and actual activity values were not significant (mean difference $=25.3 \pm 18.1 \mathrm{~min} ; \mathrm{p}=0.17$ ). The calibrated model was able to produce reasonably accurate physical activity values [60]. A similar method could be adopted in the HD population to calibrate self-report measures of physical activity.

$$
\% \mathrm{MVPA}=14.56-(\mathrm{sex} \times 0.98)-(\text { age } \times 0.84)+(1.01 \times \mathrm{PAQ})
$$

While this review concentrates on ambulatory patients undergoing HD treatment, it is also worth noting the importance of providing non-ambulatory patients ideas in which to increase physical functioning and overall wellbeing. Some prior studies suggest stretching and resistance training to increase activity in non-ambulatory patients; however, the benefit of this type of activity on patient health is still unclear. One study in particular confirmed that muscle-strengthening exercises using a Thera-band, a resistive exercise elastic band, can be a useful intervention in improving lower limb function in hemiplegic subjects [61]. Furthermore, a study by Chen et al. concluded that intradialytic, low intensity, progressive strength training utilizing resistive exercise bands and ankle weights among other tools, were safe and effective for those on dialysis [19].

The main motivation for this review is that physicians and health care workers can observe their patients only during their dialysis treatments, which constitutes only less than $10 \%$ of their daily life. Knowing more about what occurs between dialysis sessions could help improve patient care and prognosis. However, technology is rapidly evolving and this particular paper only studies literature published up to early September 2014. It is important to note that the renal field should take advantage of the development of healthcare applications for smartphones, a general trend for medicine, and patient-care as a whole. Additionally, this review focused on studies published in English literature. It is also worth acknowledging that there should be more research into discovering ways in which to mitigate all the current knowledge gaps. 
There should be a consensus on which metric is the best measure of physical activity whether it be objective (devices), subjective (self-report), or a combination of both. In addition, physical activity is rarely studied in non-ambulatory patients, a population that could potentially benefit from increased activity. Therefore, exploring methods that measure physical activity in non-ambulatory patients should be explored so that interventions to increase activity in this population can be established. Furthermore, there should be studies in the HD population that utilize the technological advancements in physical activity tracking devices mentioned in the review as culture and healthcare transition into a high-tech environment.

\section{Conclusion}

Studies have shown interdialytic activity to contribute to patient well-being and outcomes; however, there is still inadequate information about this topic. A better understanding of interventions that will lead to in- creased physical activity cannot be reached until there is a clear consensus for a universal metric to measure physical activity. Without a standard metric, it is very difficult to compare results of individual studies. There will need to be multiple components to this standard metric, since no existing tools can capture the multidimensional nature of physical activity. In addition to the traditional tools to assess physical activity, forthcoming technologies should not be disregarded as they are on the horizon of this field and renal research should strive to validate these tools amongst the HD population. Also designing studies to create baselines and establish prediction models for physical activity may be useful for providing more accurate and cost-effective activity measurements. Likewise, ways to increase activity and function among non-ambulatory HD patients are urgently needed.

\section{Disclosure Statement}

P.K. holds stock in Fresenius Medical Care.

\section{References}

1 World Health Organization: Physical activity, World Health Organization, 2014.

2 World Health Organization: Global recommendations on physical activity for health, 2010.

- 3 Yates T, Haffner SM, Schulte PJ, Thomas L, Huffman KM, Bales CW, Califf RM, Holman RR, McMurray JJ, Bethel MA, Tuomilehto J, Davies MJ, Kraus WE: Association between change in daily ambulatory activity and cardiovascular events in people with impaired glucose tolerance (NAVIGATOR trial): a cohort analysis. Lancet 2014;383: 1059-1066.

4 Foley RN, Gilbertson DT, Murray T, Collins AJ: Long interdialytic interval and mortality among patients receiving hemodialysis. $\mathrm{N}$ Engl J Med 2011;365:1099-1107.

5 Anand S, Johansen KL, Grimes B, Kaysen GA, Dalrymple LS, Kutner NG, Chertow GM: Physical activity and self-reported symptoms of insomnia, restless legs syndrome, and depression: the comprehensive dialysis study. Hemodial Int 2013;17:50-58.

6 Gordon PL, Doyle JW, Johansen KL: Postdialysis fatigue is associated with sedentary behavior. Clin Nephrol 2011;75:426-433.

7 Williams A, Stephens R, McKnight T, Dodd $S$ : Factors affecting adherence of end-stage renal disease patients to an exercise programme. Br J Sports Med 1991;25:90-93.
$>8$ Goodman ED, Ballou MB: Perceived barriers and motivators to exercise in hemodialysis patients. Nephrol Nurs J 2004;31:23-29.

-9 Moattari M, Ebrahimi M, Sharifi N, Rouzbeh $\mathrm{J}$ : The effect of empowerment on the self-efficacy, quality of life and clinical and laboratory indicators of patients treated with hemodialysis: a randomized controlled trial. Health Qual Life Outcomes 2012;10:115.

10 Hays RD, Kallich JD, Mapes DL, Coons SJ, Amin N, Carter WB, Kamberg C: Kidney Disease Quality of Life Short Form, 1997.

11 Delgado C, Johansen KL: Deficient counseling on physical activity among nephrologists. Nephron Clin Pract 2010;116:c330-c336.

12 Painter P, Clark L, Olausson J: Physical function and physical activity assessment and promotion in the hemodialysis clinic: a qualitative study. Am J Kidney Dis 2014;64:425433.

13 Cheema BS, Singh MA: Exercise training in patients receiving maintenance hemodialysis: a systematic review of clinical trials. Am J Nephrol 2005;25:352-364.

14 Painter P: Implementing exercise: what do we know? Where do we go? Adv Chronic Kidney Dis 2009; 16:536-544.

15 Johansen KL: Exercise and dialysis. Hemodialysis international. International Symposium on Home Hemodialysis 2008;12:290300 .
16 Heiwe S, Jacobson SH: Exercise training for adults with chronic kidney disease. Cochrane Database Syst Rev 2011:CD003236.

17 Avesani CM, Trolonge S, Deleaval P, Baria F, Mafra D, Faxen-Irving G, Chauveau P, Teta D, Kamimura MA, Cuppari L, Chan M, Heimburger O, Fouque D: Physical activity and energy expenditure in haemodialysis patients: an international survey. Nephrol Dial Transplant 2012;27:2430-2434.

18 Koh KP, Fassett RG, Sharman JE, Coombes JS, Williams AD: Effect of intradialytic versus home-based aerobic exercise training on physical function and vascular parameters in hemodialysis patients: a randomized pilot study. Am J Kidney Dis 2010;55: 88-99.

19 Chen JL, Godfrey S, Ng TT, Moorthi R, Liangos O, Ruthazer R, Jaber BL, Levey AS, Castaneda-Sceppa C: Effect of intra-dialytic, lowintensity strength training on functional capacity in adult haemodialysis patients: a randomized pilot trial. Nephrol Dial Transplant 2010;25:1936-1943.

20 Cheema B, Abas H, Smith B, O'Sullivan A, Chan M, Patwardhan A, Kelly J, Gillin A, Pang G, Lloyd B, Singh MF: Progressive exercise for anabolism in kidney disease (PEAK): a randomized, controlled trial of resistance training during hemodialysis. J Am Soc Nephrol 2007;18:1594-1601. 
-21 Johansen KL, Painter PL, Sakkas GK, Gordon P, Doyle J, Shubert T: Effects of resistance exercise training and nandrolone decanoate on body composition and muscle function among patients who receive hemodialysis: a randomized, controlled trial. J Am Soc Nephrol 2006;17:2307-2314.

22 Dong J, Sundell MB, Pupim LB, Wu P, Shintani A, Ikizler TA: The effect of resistance exercise to augment long-term benefits of intradialytic oral nutritional supplementation in chronic hemodialysis patients. J Ren Nutr 2011;21:149-159.

-23 Kopple JD, Wang H, Casaburi R, Fournier M, Lewis MI, Taylor W, Storer TW: Exercise in maintenance hemodialysis patients induces transcriptional changes in genes favoring anabolic muscle. J Am Soc Nephrol 2007; 18: 2975-2986.

24 Watson EL, Kosmadakis GC, Smith AC, Viana JL, Brown JR, Molyneux K, Pawluczyk IZ, Mulheran M, Bishop NC, Shirreffs S, Maughan RJ, Owen PJ, John SG, McIntyre CW, Feehally J, Bevington A: Combined walking exercise and alkali therapy in patients with CKD4-5 regulates intramuscular free amino acid pools and ubiquitin E3 ligase expression. Eur J Appl Physiol 2013;113:2111-2124.

25 Johansen KL, Sakkas GK, Doyle J, Shubert T, Dudley RA: Exercise counseling practices among nephrologists caring for patients on dialysis. Am J Kidney Dis 2003;41:171-178.

-26 Zamojska S, Szklarek M, Niewodniczy M, Nowicki M: Correlates of habitual physical activity in chronic haemodialysis patients. Nephrol Dial Transplant 2006;21:1323-1327.

27 Johansen KL, Painter P, Kent-Braun JA, Ng AV, Carey S, Da Silva M, Chertow GM: Validation of questionnaires to estimate physical activity and functioning in end-stage renal disease. Kidney Int 2001;59:1121-1127.

-28 Johansen KL, Chertow GM, Kutner NG, Dalrymple LS, Grimes BA, Kaysen GA: Low level of self-reported physical activity in ambulatory patients new to dialysis. Kidney Int 2010; 78:1164-1170.

29 Koh KP, Fassett RG, Sharman JE, Coombes JS, Williams AD: Intradialytic versus homebased exercise training in hemodialysis patients: a randomised controlled trial. BMC Nephrol 2009;10:2.

-30 Painter P, Marcus RL: Assessing physical function and physical activity in patients with CKD. Clin J Am Soc Nephrol 2013;8:861872.

- 31 Kohl Lde M, Signori LU, Ribeiro RA, Silva AM, Moreira PR, Dipp T, Sbruzzi G, Lukrafka JL, Plentz RD: Prognostic value of the sixminute walk test in end-stage renal disease life expectancy: a prospective cohort study. Clinics (Sao Paulo) 2012;67:581-586.

-32 Majchrzak KM, Pupim LB, Chen K, Martin CJ, Gaffney S, Greene JH, Ikizler TA: Physical activity patterns in chronic hemodialysis patients: comparison of dialysis and nondialysis days. J Ren Nutr 2005;15:217-224.
33 Painter P, Ward K, Nelson RD: Self-reported physical activity in patients with end stage renal disease. Nephrol Nurs J 2011;38:139-147; quiz 148.

34 Nielson R, Vehrs PR, Fellingham GW, Hager $R$, Prusak KA: Step counts and energy expenditure as estimated by pedometry during treadmill walking at different stride frequencies. J Phys Act Health 2011;8:1004-1013.

35 Robinson-Cohen C, Littman AJ, Duncan GE, Roshanravan B, Ikizler TA, Himmelfarb J, Kestenbaum BR: Assessment of physical activity in chronic kidney disease. J Ren Nutr 2013;23:123-131.

36 Johansen KL, Chertow GM, Ng AV, Mulligan K, Carey S, Schoenfeld PY, Kent-Braun JA: Physical activity levels in patients on hemodialysis and healthy sedentary controls. Kidney Int 2000;57:2564-2570.

37 Nowicki M, Murlikiewicz K, Jagodzinska M: Pedometers as a means to increase spontaneous physical activity in chronic hemodialysis patients. J Nephrol 2010;23:297-305.

38 Matsuzawa R, Matsunaga A, Wang G, Kutsuna $\mathrm{T}$, Ishii A, Abe $\mathrm{Y}$, Takagi $\mathrm{Y}$, Yoshida A, Takahira N: Habitual physical activity measured by accelerometer and survival in maintenance hemodialysis patients. Clin J Am Soc Nephrol 2012;7:2010-2016.

39 Garmin: Garmin vivofit, 2014.

40 Engadget: Nike fuelband se review: more social features, much longer battery life, 2013.

41 Virtual. Sensoria ${ }^{\circledR}$ Fitness, Sensoria ${ }^{\circledR}$ Fitness, 2013-2014.

42 Biometric shirts for performance improvement and sleep tracking. Hexoskin, Hexoskin, 2014

43 Apple: Apple Watch sport, 2014.

44 Bennington-Castro J: Electronic Skin Moves Us Closer to Cyborgs. Scientific American Global RSS, 2014

45 Boyle R: Harvard's Four-Cent Paper Accelerometer Could Make Motion Sensing Ubiquitous. Popular Science, 2011.

46 Shoaib M, Bosch S, Incel OD, Scholten H, Havinga PJ: Fusion of smartphone motion sensors for physical activity recognition. Sensors (Basel) 2014;14:10146-10176.

47 Bohm C, Stewart K, Onyskie-Marcus J, Esliger D, Kriellaars D, Rigatto C: Effects of intradialytic cycling compared with pedometry on physical function in chronic outpatient hemodialysis: a prospective randomized trial. Nephrol Dial Transplant 2014;29:1947-1955.

48 Malagoni AM, Catizone L, Mandini S, Soffritti S, Manfredini R, Boari B, Russo G, Basaglia N, Zamboni P, Manfredini F: Acute and long-term effects of an exercise program for dialysis patients prescribed in hospital and performed at home. J Nephrol 2008;21:871878.
49 Greenwood SA, Lindup H, Taylor K, Koufaki P, Rush R, Macdougall IC, Mercer TH: Evaluation of a pragmatic exercise rehabilitation programme in chronic kidney disease. Nephrol Dial Transplant 2012;27(suppl 3): iii126-iii134.

50 Kouidi E, Grekas D, Deligiannis A, Tourkantonis A: Outcomes of long-term exercise training in dialysis patients: comparison of two training programs. Clin Nephrol 2004; 61(suppl 1):S31-S38.

-51 Conn VS, Hafdahl AR, Brown SA, Brown LM: Meta-analysis of patient education interventions to increase physical activity among chronically ill adults. Patient Educ Couns 2008;70:157-172.

52 Energy intake and expenditure. British $\mathrm{Nu}$ trition Foundation, 2009

53 Zhang M, Kim JC, Li Y, Shapiro BB, Porszasz J, Bross R, Feroze U, Upreti R, Martin D, Kalantar-Zadeh K, Kopple JD: Relation between anxiety, depression, and physical activity and performance in maintenance hemodialysis patients. J Ren Nutr 2014;24:252-260.

54 Morishita Y, Kubo K, Miki A, Ishibashi K, Kusano E, Nagata D: Positive association of vigorous and moderate physical activity volumes with skeletal muscle mass but not bone density or metabolism markers in hemodialysis patients. Int Urol Nephrol 2014;46:633639

55 Grip strength. Wikipedia Foundation, 2014.

56 Janssen I, Heymsfield SB, Ross R: Low relative skeletal muscle mass (sarcopenia) in older persons is associated with functional impairment and physical disability. J Am Geriatr Soc 2002;50:889-896

57 Delgado C, Johansen KL: Barriers to exercise participation among dialysis patients. Nephrol Dial Transplant 2012;27:11521157.

58 Plasqui G, Westerterp KR: Physical activity assessment with accelerometers: an evaluation against doubly labeled water. Obesity (Silver Spring) 2007;15:2371-2379.

59 Prince SA, Adamo KB, Hamel ME, Hardt J, Connor Gorber S, Tremblay M: A comparison of direct versus self-report measures for assessing physical activity in adults: a systematic review. Int J Behav Nutr Phys Act 2008;5: 56.

60 Saint-Maurice PF, Welk GJ, Beyler NK, Bartee RT, Heelan KA: Calibration of self-report tools for physical activity research: the Physical Activity Questionnaire (PAQ). BMC Public Health 2014;14:461

61 Han SS, Her JJ, Kim YJ: Effects of muscle strengthening exercises using a Thera Band on lower limb function of hemiplegic stroke patients. Taehan Kanho Hakhoe Chi 2007;37: 844-854.

62 Make fitness a lifestyle with flex ${ }^{\mathrm{TM}}$. Fitbit ${ }^{\circledR}$ Flex $^{\mathrm{TM}}, 2014$

63 Buy up by jawbone ${ }^{\mathrm{TM}}, 2014$ 